\title{
Brane-World Dark Matter
}

\author{
J. A. R. Cembranos, A. Dobado, and A. L. Maroto \\ Departamento de Física Teórica, Universidad Complutense de Madrid, 28040 Madrid, Spain
} (Received 12 February 2003; revised manuscript received 10 April 2003; published 17 June 2003)

\begin{abstract}
We show that, in the context of brane-world scenarios with low tension $\tau=f^{4}$, massive brane fluctuations (branons) are natural dark matter candidates. We calculate the present abundances for both hot (warm) and cold branons in terms of the branon mass $M$ and the tension scale $f$. The results are compared with the current experimental bounds on these parameters. We also study the prospects for their detection in direct search experiments and comment on their characteristic signals in the indirect ones.
\end{abstract}

DOI: 10.1103/PhysRevLett.90.241301

PACS numbers: 95.35.+d, 11.10.Kk, 11.25.Wx

One of the most important open problems in astrophysics and cosmology is to identify the nature of dark matter. It has been known for a long time that the luminous matter observed in spiral galaxies is insufficient to explain their rotation curves. The existence of dark halos was proposed as a possible solution for the discrepancy (see [1] and references therein), although, at present, numerical simulations of the formation of such halos appear inconsistent with observations. On the other hand, different estimations of the total matter density in the universe from large scale motions, virial masses or cluster abundances, and the more recent type Ia supernovae and cosmic microwave background anisotropy observations agree in a value $\Omega_{M}=0.27 \pm 0.04[2,3]$, which is much larger than the value of the total luminous mass density in the universe $\Omega_{\text {lum }} h=0.006-0.002$. In addition, the big bang nucleosynthesis (BBN) and Wilkinson microwave anisotropy probe (WMAP) results for the total baryonic content $\Omega_{B} h^{2}=0.0224 \pm 0.0009$ imply that most of the matter in the universe is dark and nonbaryonic (see [2-4] and references therein).

A possible explanation of this puzzle is that the dominant component of dark matter consists of some nonrelativistic (cold) stable and weakly interacting massive particles (WIMP) which decoupled from radiation early enough so that their relic abundances are important today. The possibility that the universe is dominated by hot dark matter seems to conflict with numerical simulations of structure formation. Thus, the only potential candidates within the known particles would be massive neutrinos. However, a detailed analysis has excluded the three light and even one additional heavy fourth generation of Majorana or Dirac neutrinos. This fact has led to the search of cold dark matter candidates beyond the Standard Model (SM) [4].

There are two main such particles studied in the literature. On one hand are axions which strictly speaking cannot be considered as WIMPs since they are very light and produced nonthermally. On the other hand, we have the lightest supersymmetric particle, which can be identified with a neutralino in most of the supersymmetric models. The latter is probably the most studied and best theoretically motivated dark matter candidate [4]. However, the large number of free parameters in supersymmetric theories make their predictions extremely model dependent. More recently, the existence of large extra dimensions has been proposed as a new setting for a possible solution to the hierarchy problem [5]. In this scenario, the SM fields are forced to live on a threedimensional hypersurface (brane), whereas gravity is able to propagate on the higher $D=4+N$ dimensional bulk space. In this brane-world scenario (BWS), the fundamental scale of gravity is not the Planck scale $M_{P}$ but another scale $M_{D}$. Although in the original ArkaniHamed-Dimopoulos-Dvali model [5], $M_{D}$ is supposed to be not too much larger than the electroweak scale, recently brane cosmology models have arisen in which $M_{D}$ is much larger than the $\mathrm{TeV}$ ([6] and references therein). In this work we will consider the case of a general BWS without assuming any particular value for $M_{D}$.

In these models, gravitons propagating through the bulk space give rise to a Kaluza-Klein (KK) tower of massive gravitons on the brane. These $\mathrm{KK}$ gravitons couple to the energy-momentum tensor of the SM fields $T_{\mathrm{SM}}^{\mu \nu}$ and could be produced under the appropriate circumstances as real or virtual particles. Another important effect that is expected in the BWS is the presence of brane fluctuations since rigid objects do not exist in relativistic theories. In other words, the brane should have some finite tension $\tau=f^{4}$. When these oscillations are taken into account, two new effects appear [7]. First of all, we have to introduce new fields which, for a homogeneous extra space, essentially represent the position of the brane in the bulk space $\left[x^{\mu}, y^{\alpha} \simeq \pi^{\alpha}(x) / f^{2}\right]$. The $\pi^{\alpha}(x)$ fields are the Goldstone bosons (GB) corresponding to the spontaneous symmetry breaking (SSB) of the translational invariance produced by the presence of the brane (branons). It has been shown [7] that, when these branons are properly taken into account, the coupling of the SM particles to any bulk field is exponentially suppressed by a factor $\exp \left[-M_{\mathrm{KK}}^{2} M_{D}^{2} /\left(8 \pi^{2} f^{4}\right)\right]$, where $M_{\mathrm{KK}}$ is the mass of the corresponding $\mathrm{KK}$ mode. As a consequence, if the 
tension scale $f$ is much smaller than the fundamental scale $M_{D}$, i.e., $f \ll M_{D}$, the KK modes decouple from the SM particles. Therefore, for flexible enough branes, the only relevant degrees of freedom at low energies in the BWS are the SM particles and the branons. Similarly to other GBs, branons are expected to be nearly massless and weakly interacting at low energies. Nevertheless, in general, translational invariance in the extra dimensions is not necessarily an exact symmetry, and some branon mass $M$ is expected from such explicit symmetry breaking as shown in $[8,9]$. This is similar to what happens to pions which are the GB corresponding to the SSB of the chiral symmetry of low-energy strong interactions. As gravitons do, branons couple to $T_{\mathrm{SM}}^{\mu \nu}$; however, in this case, the lowest order effective Lagrangian is [9]

$$
\begin{aligned}
\mathcal{L}_{B r}= & \frac{1}{2} g^{\mu \nu} \partial_{\mu} \pi^{\alpha} \partial_{\nu} \pi^{\alpha}-\frac{1}{2} M^{2} \pi^{\alpha} \pi^{\alpha} \\
& +\frac{1}{8 f^{4}}\left(4 \partial_{\mu} \pi^{\alpha} \partial_{\nu} \pi^{\alpha}-M^{2} \pi^{\alpha} \pi^{\alpha} g_{\mu \nu}\right) T_{\mathrm{SM}}^{\mu \nu} .
\end{aligned}
$$

We see that branons always interact by pairs, they are stable and difficult to detect, since their interactions are suppressed by the tension scale $f$, and they are expected to be massive. Thus, we arrive at the conclusion that the massive oscillations of the brane are natural candidates to dark matter in the BWS where $f \ll M_{D}$. The dark matter problem has been considered in this scenario from a different point of view in [10] and also in models with universal extra dimensions [11].

In order to calculate the thermal relic branon abundance, we will use the standard techniques given in [12] in two limiting cases, either relativistic (hot) or nonrelativistic (cold) branons at decoupling. The evolution of the number density $n_{\alpha}$ of branons $\pi^{\alpha}, \alpha=1, \ldots, N$ interacting with SM particles in an expanding universe is given by the Boltzmann equation:

$$
\frac{d n_{\alpha}}{d t}=-3 H n_{\alpha}-\left\langle\sigma_{A} v\right\rangle\left[n_{\alpha}^{2}-\left(n_{\alpha}^{e q}\right)^{2}\right],
$$

where $\sigma_{A}=\sum_{X} \sigma\left(\pi^{\alpha} \pi^{\alpha} \rightarrow X\right)$ is the total annihilation cross section of branons into SM particles $X$ summed over all final states. The $-3 H n_{\alpha}$ term, with $H$ the Hubble parameter, takes into account the dilution of the number density due to the universe expansion. These are the only terms which could change their number density to the leading order. In fact, branons do not decay into other particles and since they interact always by pairs the conversions such as $\pi^{\alpha} X \rightarrow \pi^{\alpha} Y$ do not change their number. Notice that we are considering the low-energy effective Lagrangian in (1) and assuming for simplicity that all the branons are degenerate. Accordingly, each branon species evolves independently, and in the following we will drop the $\alpha$ index. The total branon density will be just $N$ times that of a single branon. The $\left\langle\sigma_{A} v\right\rangle$ term denotes the thermal average of the total annihilation cross section times the relative velocity. From (1), it includes, to leading order, annihilations into all the SM particle-antiparticle pairs. If the universe temperature is above the QCD phase transition $\left(T>T_{c}\right)$, we consider annihilations into quark-antiquark and gluons pairs. If $T<T_{c}$, we include annihilations into light hadrons. For the sake of definiteness, we have taken a critical temperature $T_{c} \simeq 170 \mathrm{MeV}$ and a Higgs boson mass $m_{H} \simeq$ $125 \mathrm{GeV}$, although the final results are not very sensitive to the concrete value of these parameters.

Defining the new variable $x=M / T$, one finds from (2) that, below the freeze-out temperature $x_{f}$ for which the annihilation rate $\Gamma_{A}=n_{e q}\left\langle\sigma_{A} v\right\rangle$ equals the expansion rate $H$, branons are decoupled from the thermal bath and their abundance remains frozen relative to the entropy density. We will denote $g_{\text {eff }}(T)$ and $h_{\text {eff }}(T)$ the effective relativistic degrees of freedom for energy and entropy densities, respectively, at temperature $T$. In order to calculate the decoupling temperature for hot relics, it is a good approximation to use the condition $\Gamma_{A}\left(x_{f}\right) \simeq$ $H\left(x_{f}\right)$. From the Friedmann equation in a radiation dominated universe, we have $H(x) \simeq 1.67 g_{\text {eff }}^{1 / 2} M^{2} /\left(x^{2} M_{P}\right)$. On the other hand, expanding $\Gamma_{A}(x)$ for $x \ll 3$ and neglecting $M$, we find $\Gamma_{A}^{\gamma}(T) \simeq 16 \pi^{9} T^{9} /\left[297675 \zeta(3) f^{8}\right]$ for photons and $\Gamma_{A}^{\nu}(T)=\Gamma_{A}^{\gamma}(T) / 4$ for neutrinos. For massive particles we cannot give closed expressions. Once we know $x_{f}$, the corresponding fraction of energy density today in the form of relics is given by

$$
\Omega_{B r} h^{2} \simeq \frac{7.83 \times 10^{-2}}{h_{\mathrm{eff}}\left(x_{f}\right)} \frac{M}{\mathrm{eV}} .
$$

For cold relics the calculation of the decoupling temperature is more complicated. The well-known result is

$$
x_{f}=\ln \left(\frac{0.038 c(c+2) M_{P} M\left\langle\sigma_{A} v\right\rangle}{g_{\mathrm{eff}}^{1 / 2} x_{f}^{1 / 2}}\right),
$$

where $c \simeq 0.5$ is obtained from the numerical resolution of the Boltzmann equation. The above equation can be solved iteratively. The matter density can be written as

$$
\Omega_{B r} h^{2} \simeq 8.766 \times 10^{-11} \mathrm{GeV}^{-2} \frac{x_{f}}{g_{\mathrm{eff}}^{1 / 2}}\left(\sum_{n=0}^{\infty} \frac{c_{n}}{n+1} x_{f}^{-n}\right)^{-1},
$$

where we have expanded $\left\langle\sigma_{A} v\right\rangle$ in powers of $x^{-1}$ as $\left\langle\sigma_{A} v\right\rangle=\sum_{n=0}^{\infty} c_{n} x^{-n}$. In the case of photons, the first nonvanishing coefficient is $c_{2}^{\gamma}=68 M^{6} /\left(15 f^{8} \pi^{2}\right)$ and for massless neutrinos $c_{2}^{\nu}=c_{2}^{\gamma} / 4$ ( $d$ wave annihilation), whereas for nonconformal matter we also have $s$ and $p$ wave annihilation $\left(c_{0}, c_{1} \neq 0\right)$. The corresponding expressions are more complicated and will be given elsewhere. We have performed all the expansions up to $\mathcal{O}\left(x^{-2}\right)$. Coannihilation effects are absent in this case since there are no slightly heavier particles which eventually could decay into the lightest branon. Also, in order to avoid the problems of the Taylor expansion near SM thresholds, we have taken branon masses sufficiently 
separated from SM particle masses where the usual treatment is adequate [12]. Such treatment is known to introduce errors of the order of $10 \%$ in the relic abundances.

Branons could be responsible for the observed cosmological dark matter density provided $\Omega_{B r} h^{2}=0.129-$ 0.095 at the $95 \%$ C.L. which corresponds to $\Omega_{B r}=$ $0.23 \pm 0.08$ and $h=0.79-0.65$ [3]. In Fig. 1, we have plotted these curves for hot and cold branons in the $f-M$ plane for one single branon. For $N$ types of branons, the corresponding abundances are simply $N$ times larger. In fact, the contribution of branons to $g_{\text {eff }}$ in (5) is negligible in the case of cold relics. For hot relics such contribution in (3) has been taken into account, although it is very small in the interesting regions. Concerning the freezeout temperature, the results on the cold dark matter curve range from $x_{f} \simeq 9$ for $M=10^{-5} \mathrm{GeV}$ to $x_{f} \simeq 31$ for $M=$ $10^{6} \mathrm{GeV}$. In the hot relics case, a very good approximation is given by $\log \left(T_{f} / \mathrm{GeV}\right) \simeq(8 / 7) \log (f / \mathrm{GeV})-3.2$.

Pure hot dark matter models are disfavored at present because relativistic matter free streams from overdense into underdense regions preventing structures from growing below the so-called free-streaming scale given by [13]: $\lambda_{F S} \simeq 0.2\left(\Omega_{B r} h^{2}\right)^{1 / 3}(\mathrm{keV} / M)^{4 / 3} \mathrm{Mpc}$. In the hot branons curve in Fig. 1, the masses in the allowed region are in the range $M=85-177 \mathrm{eV}$, which corresponds to $\lambda_{F S} \simeq 2.4-1.0 \mathrm{Mpc}$. Such scales are much smaller than those in neutrino dark matter models and, in addition, since these branons decouple much earlier than neutrinos do, their corresponding temperatures are also lower; i.e., they could be considered rather as warm dark matter (WDM) candidates [13] from the point of view of structure formation. However, a WDM dominated universe seems to be also disfavored by the recent observations

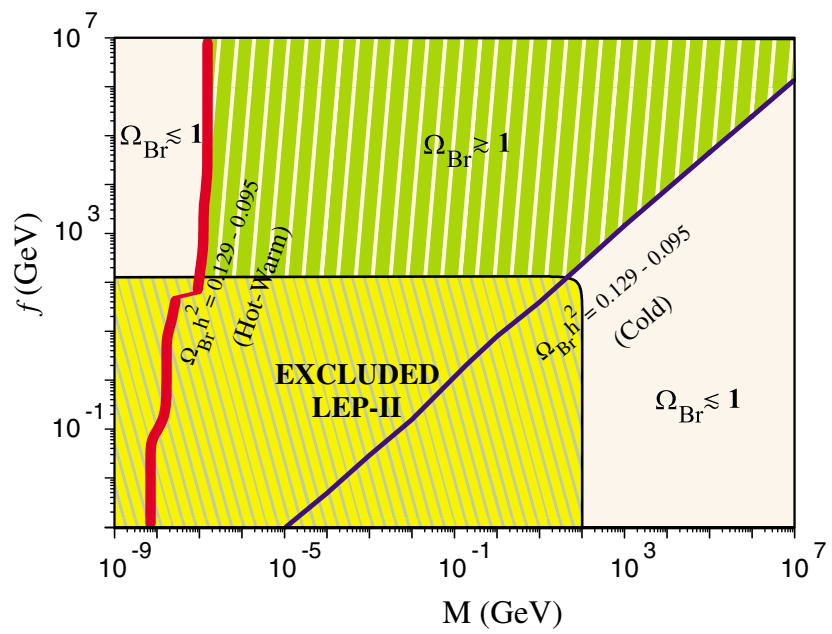

FIG. 1 (color online). Relic abundance for a model with one branon $N=1$. The line on the left is the $\Omega_{B r} h^{2}=0.129-$ 0.095 curve for hot-warm relics. The line on the right corresponds to cold relics. The lower striped area is the estimated excluded region by single-photon processes at CERN $e^{+} e^{-}$ collider LEP-II [9] and the upper area is also excluded by branon overproduction. of WMAP [3]. On the other hand, the presence of relativistic branons during $\mathrm{BBN}$ could change the expansion rate of the universe, spoiling the predictions of the light element abundances [12]. However, the BBN limits on the number of branons contributing to $g_{\text {eff }}$ are not very constraining. In fact, imposing the conservative bound $g_{\text {eff }}\left(T_{B B N} \sim 1 \mathrm{MeV}\right) \lesssim 12.5$ [12], we find $N \lesssim 18$ for branons which decoupled before the QCD phase transition, corresponding to $f \geqslant 60 \mathrm{GeV}$.

Let us clarify the main assumptions used thus far in the work. We are considering models with $f \ll M_{D}$, and also assuming that the evolution of the universe is standard up to a temperature around $f$. Indeed, this is the case of realistic brane cosmology models [6]. Therefore, we have taken the conservative bound $f>1 \mathrm{MeV}$, so that BBN is not affected. Moreover, the effective Lagrangian (1) is valid only at low energies relative to $f$. We have checked that our calculations are consistent with these assumptions since the decoupling temperatures are always smaller than $f$ in the allowed regions in Fig. 1.

Brane fluctuations could be not only candidates for the cosmological dark matter, but also they could make up the galactic halo and explain the local dynamics. In such a case, they could be detected in direct search experiments from the energy transfer in elastic collisions with nuclei of a suitable target. The appropriate quantity to be

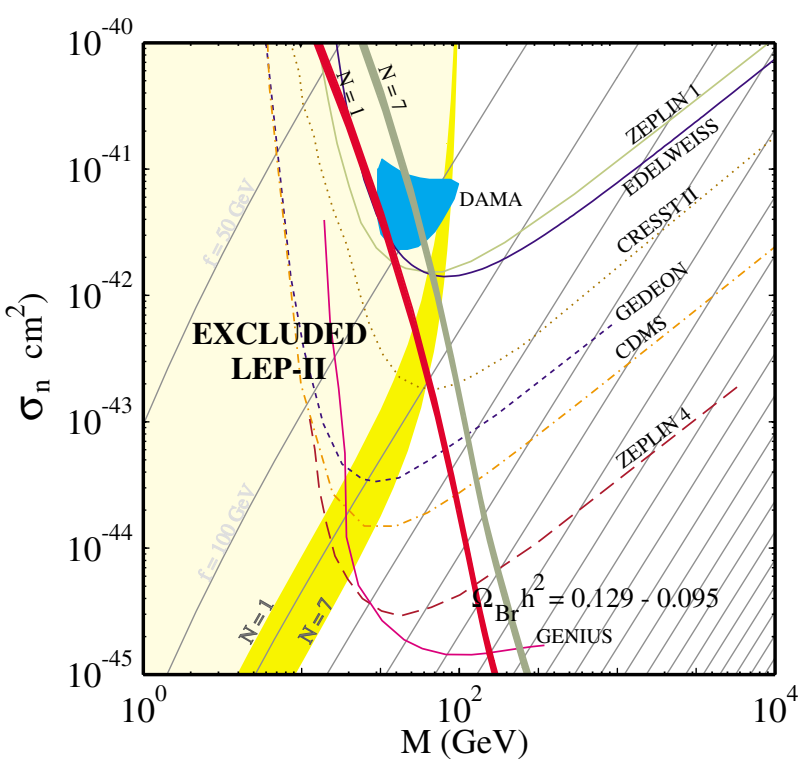

FIG. 2 (color online). Elastic branon-nucleon cross section $\sigma_{n}$ in terms of the branon mass. The two thick lines correspond to the $\Omega_{B r} h^{2}=0.129-0.095$ curve for cold branons in Fig. 1 with $N=1$ (left) and $N=7$ (right). The shaded areas on the left are the previous LEP-II exclusion regions [9], also for $N=$ 1,7. The solid lines correspond to the current limits on the spin-independent cross section from direct detection experiments: ZEPLIN1 [14], DAMA [15], and EDELWEISS [16]. The discontinuous lines are the projected limits for CRESST [17], GEDEON [18], CDMS [19], ZEPLIN4 [20], and GENIUS [21] (limits obtained from [22]). 
compared with the experimental results is not the elastic branon-nucleus cross section $\sigma$, but the differential cross section per nucleon at zero-momentum transfer $\sigma_{n}$, which is defined by [4]

$$
\frac{d \sigma}{d|q|^{2}}=\frac{\sigma_{n} A^{2} F^{2}(|q|)}{4 v^{2} \mu^{2}},
$$

where $\mu=M m /(M+m), F(|q|)$ is a nuclear form factor with the normalization $F(0)=1, m \simeq 939 \mathrm{MeV}$ is the nucleon mass, $v$ is the relative velocity, and $A$ is the mass number of the nucleus. In the limit in which the momentum transfer goes to zero, we can consider the nucleons as pointlike particles. In this case, it is possible to calculate the branon-nucleon cross section $\sigma_{n}$ from (1) just considering the nucleon as a Dirac fermion of mass $m$ :

$$
\sigma_{n}=\frac{9 M^{2} m^{2} \mu^{2}}{64 \pi f^{8}} \text {. }
$$

In fact, this quantity does not depend on the type of particle which couples to the branon, but only on its mass. This can be seen from (1) since in this limit, branons couple only to the $T^{00}$ component.

The results of our analysis are shown in Fig. 2. Lines of constant $f$ with $50 \mathrm{GeV}$ separation are shown for reference. The area on the left of the $\Omega_{B r} h^{2}=0.129-0.095$ curves is excluded by branon overproduction, but the right portion is compatible with observations and will be explored in future experiments. Such a region corresponds to $f \gtrsim 120 \mathrm{GeV}$ and $M \gtrsim 40 \mathrm{GeV}$.

Another interesting possibility is to detect branons indirectly. Their annihilations in the galactic halo can give rise to pairs of photons or $e^{+} e^{-}$which could be detected by $\gamma$-ray telescopes such as MAGIC or GLAST or antimatter detectors. Annihilation of branons trapped in the center of the Sun or the earth can give rise to high-energy neutrinos which could be detectable by high-energy neutrino telescopes such as AMANDA, IceCube, or ANTARES (see, for example, [11]). Because annihilations of nonrelativistic branons into conformal matter are $d$-wave suppressed, the most relevant contribution will come from the secondary leptonic decays of ultrarelativistic $Z$ or $W$ bosons (in the case $M \gg M_{Z}$ ). These processes will be characterized by the presence of peaks around one-half of the branon mass in the leptonic or neutrino spectra. In the case of photons, softer peaks will be present at lower energies and therefore their detection will be more difficult. The hadronic decays will give rise to relatively smeared spectra at lower energies. Detailed results will be presented elsewhere.

Throughout this Letter, we have assumed that branons were in thermal equilibrium with radiation at some point in the history of the universe. If this is not the case, branons could still be produced nonthermally, very much in the same way as axionic dark matter [23]. In fact, for very light branons, the energy density produced by this mechanism could be cosmologically important.
In conclusion, we have proposed branons as natural dark matter candidates in the BWS with low tension. Our results show that, in a certain range of the parameters $f$ and $M$, their relic abundances could explain the missing mass problem, and that such parameter regions will be explored in future direct detection experiments.

This work has been partially supported by the DGICYT (Spain) under Projects No. FPA 2000-0956 and No. BFM2000-1326.

[1] J. Binney and S. Tremaine, Galactic Dynamics (Princeton University Press, Princeton, NJ, 1987).

[2] Particle Data Group, K. Hagiwara et al., Phys. Rev. D 66, 010001 (2002).

[3] D. N. Spergel et al., astro-ph/0302209.

[4] K. Griest and M. Kamionkowski, Phys. Rep. 333, 167 (2000).

[5] N. Arkani-Hamed, S. Dimopoulos, and G. Dvali, Phys. Lett. B 429, 263 (1998); N. Arkani-Hamed, S. Dimopoulos, and G. R. Dvali, Phys. Rev. D 59, 086004 (1999); I. Antoniadis, Phys. Lett. B 246, 377 (1990).

[6] D. Langlois, in Proceedings of YITP Workshop: Braneworld: Dynamics of Space-time Boundary, Kyoto, Japan [Prog. Theor. Phys. Suppl. 148, 181 (2003)].

[7] M. Bando et al., Phys. Rev. Lett. 83, 3601 (1999); R. Sundrum, Phys. Rev. D 59, 085009 (1999).

[8] A. Dobado and A. L. Maroto, Nucl. Phys. B592, 203 (2001).

[9] J. Alcaraz, J. A. R. Cembranos, A. Dobado, and A. L. Maroto, Phys. Rev. D 67, 075010 (2003).

[10] G. Dvali, Phys. Lett. B 459, 489 (1999); N. ArkaniHamed et al., J. High-Energy Phys. 0012 (2000) 010.

[11] H.-C. Cheng, J. L. Feng, and K.T. Matchev, Phys. Rev. Lett. 89, 211301 (2002); G. Servant and T. M. P. Tait, Nucl. Phys. B650, 391 (2003).

[12] E.W. Kolb and M.S. Turner, The Early Universe (Addison-Wesley, Reading, MA, 1990); M. Srednicki, R. Watkins, and K. A. Olive, Nucl. Phys. B310, 693 (1988); P. Gondolo and G. Gelmini, Nucl. Phys. B 360, 145 (1991).

[13] P. Colin, V. Avila-Reese, and O. Valenzuela, Astrophys. J. 542, 622 (2000).

[14] N. J. T. Smith, at the 4th International Workshop on the Identification of Dark Matter, IDM2002, York, UK, 2002.

[15] R. Bernabei et al., Phys. Lett. B 480, 23 (2000).

[16] A. Benoit et al., Phys. Lett. B 545, 43 (2002).

[17] M. Altmann et al., astro-ph/0106314.

[18] A. Morales et al., Phys. Lett. B 532, 8 (2002).

[19] R.W. Schnee et al., Phys. Rep. 307, 283 (1998).

[20] H. Wang, at the 5th International UCLA Symposium DM2002, Marina del Rey, 2002.

[21] L. Baudis et al., Phys. Rep. 307, 301 (1998).

[22] R. Gaitskell and V. Mandic, http://dmtools.berkeley.edu

[23] J. Preskill, M. B. Wise, and F. Wilczek, Phys. Lett. B 120, 127 (1983); J. A. Frieman and A. H. Jaffe, Phys. Rev. D 45, 2674 (1992). 